\title{
Acoustic Emission from Organic Martensites
}

\author{
Manas K. Panda, ${ }^{\mathrm{a}, \dagger}$ Martin Etter, ${ }^{\mathrm{b}}$ Robert E. Dinnebier, ${ }^{\mathrm{c}}$ Panče Naumov ${ }^{\mathrm{a}, \star}$ \\ Dedicated to Professor Bojan Šoptrajanov, on the occasion of his $80^{\text {th }}$ birthday
}

\begin{abstract}
In salient effects, still crystals of solids that switch between phases acquire a momentum and are autonomously propelled due to rapid release of elastic energy accrued during a latent structural transition induced by heat, light or mechanical stimulation. Herein we report that when the mechanical reconfiguration is induced by change of temperature in thermosalient crystals, bursts of detectable acoustic waves are generated prior to their self-actuation. The results provide compelling evidence that the thermosalient transitions in organic and organic-containing crystals are molecular analogues of the martensitic transitions in some metals and metal alloys such as steel and shape memory alloys. Within a broader context, the results reveal that akin to metallic bonding, the intermolecular interactions in molecular solids are capable of gradual accrual and sudden release of substantial amount of strain during anisotropic thermal expansion, followed by a rapid transformation of the crystal packing in a diffusionless, non-displacive transition.
\end{abstract}

Thermosalient crystals are a rare class of molecular solids which, when transitioning between two phases, are capable of autonomous actuation whereby they spontaneously acquire a momentum and propel themselves. ${ }^{1-10}$ This phenomenon, termed thermosalient effect, is a striking demonstration of a fundamental process of energy transduction-the conversion of disordered molecular motion (heat) into an ordered motion (movement) by collective and concerted action of molecules in the ordered solid state. The motion of crystals can also be triggered by light, following a photochemical reaction (photosalient effect), ${ }^{11-14}$ or by mechanical stimulation of a prestrained crystal (mechanosalient effect). ${ }^{15}$ The autonomous motility of these solids is due to rapid release of elastic energy that has been accrued during an anisotropic thermal expansion preceding the transition. Despite the potentials that these materials could have for fast transduction of heat directly into motion or work, the short time scales of their transitions and the frequent disintegration with explosive outcome have precluded elucidation of the underlying mechanisms and quantification of their kinetics. Common features such as rapid progression of the phase front (the habit plane), ${ }^{1}$ pronouncedly anisotropic thermal expansion preceding the transition, ${ }^{1,8}$ preservation of crystal symmetry, ${ }^{1}$ and conformational and packing similarity between the two phases,,$^{1,3,7,8}$ hint at analogy between the transitions in these organic-containing molecular materials and the martensitic transitions-diffusionless,

\footnotetext{
[a] M. K Panda, P. Naumov, New York University Abu Dhabi, PO Box 129188, Abu Dhabi, UAE.

E-mail: pance.naumov@nyu.edu

[b] M. Etter, Deutsches Elektronen Synchrotron (DESY), FS-PE, P02.1, Notkestr. 85, 22607 Hamburg, Germany

[c] R. E. Dinnebier, Max Planck Institute for Solid State Research, Heisenbergstrasse 1, D-70569 Stuttgart, Germany

† Present address: Photo Science \& Photonics Section, Chemical Science and Technology Division, CSIR-National Institute for Interdisciplinary Science \& Technology, Trivandrum 695019, India

Supporting information for this article is given via a link at the end of the document.
}

displacive first-order phase transformations observed with steel and some shape-memory alloys. However, due to the extremely fast rates and frequent disintegration, direct experimental evidence of this hypothesis has not been provided yet. ${ }^{16-28}$

We hypothesized that the sudden release of the accrued elastic strain in thermosalient crystals, reflected in extraordinarily high velocities of the progressing habit plane could generate detectable acoustic waves. In addition to sudden release of accumulated strain ${ }^{29,30}$ elastic waves can evolve by dislocations in plastic deformation, ${ }^{31-34}$ crack formation, ${ }^{35}$ anisotropic thermal expansion ${ }^{36,37}$ and rapid molecular rearrangement. ${ }^{38-42}$ However, unlike the continuous acoustic emission that occurs during plastic deformation with slow progression of dislocations, the response from the martensitic transitions is discontinuous. Being generated in avalanches akin to seismic waves that precede an earthquake, the intensity of the acoustic bursts is several orders of magnitude higher than that of the continuous emission. Herein, we provide direct evidence that in addition to only one brief previously reported case ${ }^{5}$ the phase transitions in thermosalient crystals are generally associated with outbursts of elastic energy that translate into acoustic waves. The energy, intensity, and time scale of the thermoacoustic response is scrutinized with the mechanism of the thermosalient transition. The associated strong waves carry important information on the dynamics and mechanism of transformation. ${ }^{43}$ Not only that the results provide deeper understanding of the mechanistic and kinetic profile of the thermosalient phenomenon, but in addition to the previous conclusions, they confirm that the thermosalient materials are molecular analogues of the inorganic martensites and that the molecular solids can behave similar to metals and alloys.

To verify the hypothesis, we selected the $L$ enantiomer of pyroglutamic acid (L-PGA) whose crystals undergo thermosalient phase transition from form $\alpha$ to form $\beta$ at $338.2-340.4 \mathrm{~K}$ on heating, and from $\beta$ to $\alpha$ at $328.7-326.9 \mathrm{~K}$ on cooling, based on DSC measurements of single crystals (the transition temperature varies with crystal size and multiplicity). ${ }^{2}$ The variabletemperature powder diffraction patterns collected using synchrotron X-ray radiation (Figure 1) confirm that the transition occurs in powders and crystals of the two enantiomers of PGA ( $L$ and D) which have identical phase transition temperatures on heating as well as on cooling. Thus, only the $L$ enantiomer and the racemate were considered for the acoustic measurements. The acoustic emission is a sensitive technique to detect transient microscopic dynamic events by recording the elastic waves generated from a source of elastic waves in the crystal bulk (for definition of the related variables and the terminology, see Figure $2 \mathrm{a}$ and the discussion below).

To record the emission, 5 single crystals of L-PGA were studied. Each single crystal was in turn heated between two copper plates, one of which was coupled to a piezoelectric acoustic sensor (Figure 2b, for details, see the Experimental section). The acoustic waves were transduced by the medium from their origin to the surface of the crystal where they were detected by a piezoelectric transducer and a sensor. Each crystal was heated 

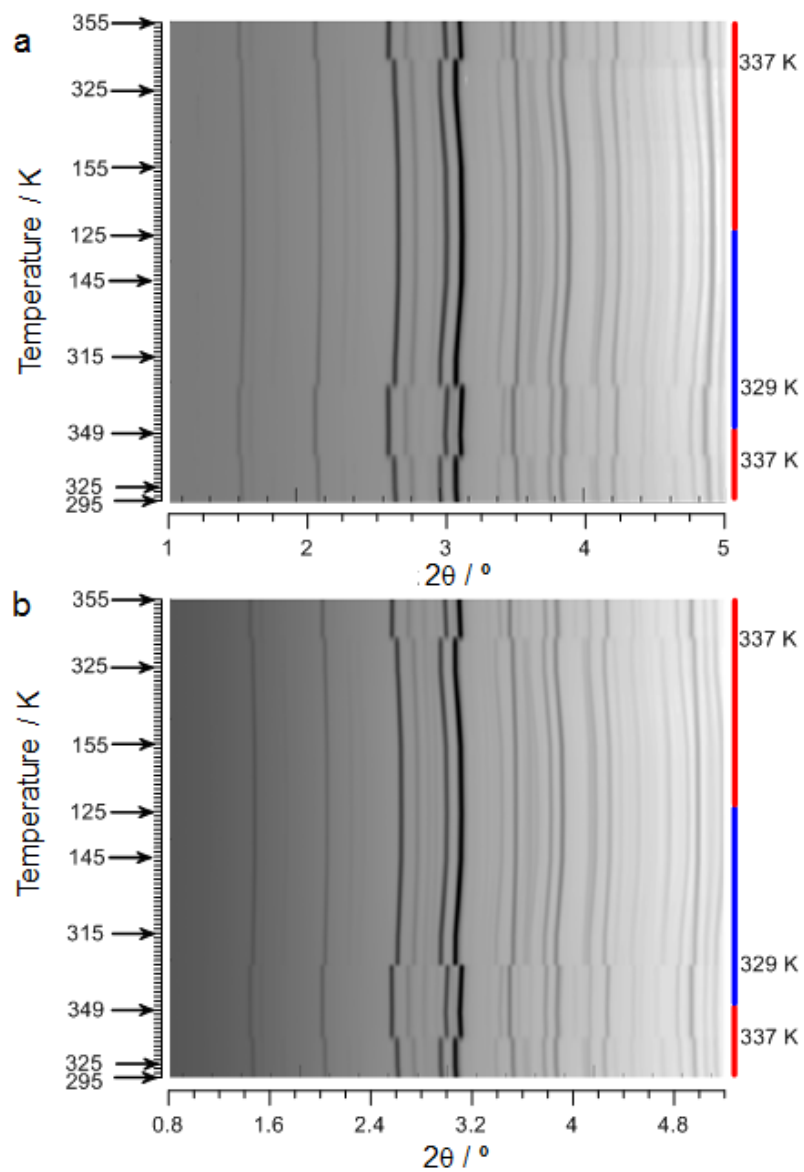

Figure 1. Top view of the surface plot of the variable-temperature $X$-ray powder diffraction patterns of L-PGA (a) and D-PGA (b) generated with the Powder3D program. ${ }^{45}$ The dependence of the powder patterns of a run on the scattering angle and scan number are shown (one scan was taken over $180 \mathrm{~s}$ ).

from room temperature to above its phase transition temperature and cooled to room temperature while recording the acoustic emission.

The most striking features in the acoustic emission are the distinct and clearly detectable acoustic bursts that appear only in the temperature region of the phase transition (Figure 3). When a single crystal of L-PGA of size $20 \times 10 \times 6 \mu \mathrm{m}^{3}$ was heated from $298 \mathrm{~K}$ to $356 \mathrm{~K}$, no acoustic emission could be detected up to 337 $\mathrm{K}$, suggesting that the crystals do not undergo any sizable lattice deformation. The anisotropic thermal expansion of the unit cell ${ }^{2}$ is insufficient to trigger any substantial lattice deformation that could generate acoustic wave above the $40 \mathrm{~dB}$ threshold. As shown in Figure $3 a-d$, however, sudden acoustic bursts with three distinct hits appear in the temperature range 338.2-339.1 $\mathrm{K}$ which corresponds to the thermosalient phase transition of the L-PGA crystal. The amplitudes of the hits were 92, 50, $58 \mathrm{~dB}$ with average frequencies of 79,21 and $87 \mathrm{kHz}$, and their rise times (time required for a signal to reach to maximum amplitude) were $65,142,159 \mu \mathrm{s}$, respectively (Table S1, Supporting Information).

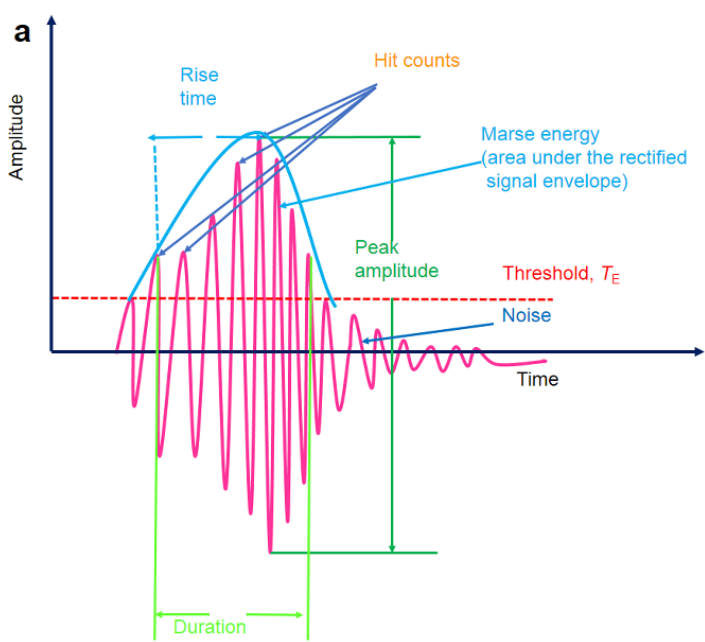

b

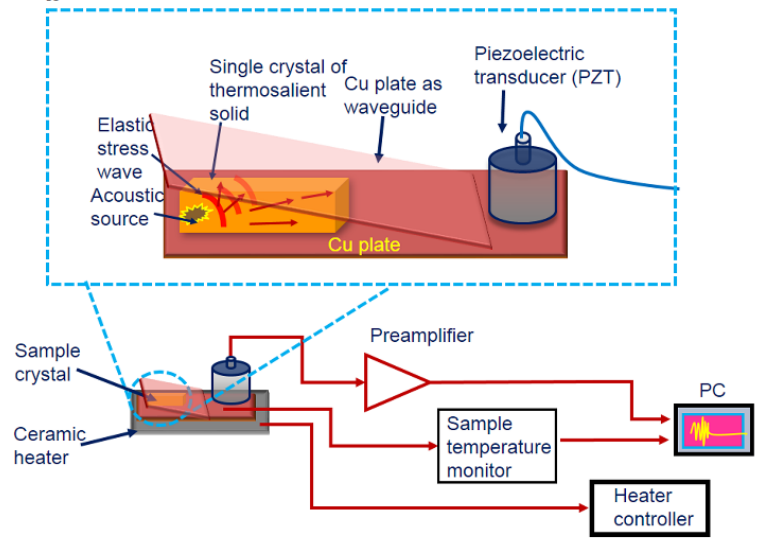

Figure 2. (a) Definition of variables and terminology related to the acoustic emission. (b) Schematic diagram of the setup used to record the acoustic emission.

The hits correspond to absolute energies $6.2 \times 10^{6}, 0.4 \times 10^{3}$, and $3.6 \times 10^{3}$ aJ respectively. Further heating up to $356 \mathrm{~K}$ did not result in additional emission hits. The "hits" are related to the progression of a disturbance as a result of rapid phase transition in a crystal domain in form of a longitudinal pressure wave (Figure 2a). The number of hits of a wave corresponds to the times the disturbance exceeds an arbitrary threshold value. A hit count is the number of times the pressure increases above the threshold. The signal strength is the intensity of each hit, the amplitude is the difference between the intensity of the strongest positive and strongest negative value of the signal, rise time is the time it takes for the signal since the first time it crosses the threshold to reach maximum intensity.

The acoustic emission from the L-PGA crystal is representative of the elastic stress wave that originates from rapid microstructural perturbation caused by supramolecular packing changes during the thermosalient transition. Notably, the strength and absolute energy of the first hit is much higher in magnitude than the subsequent hits, while its risetime is much shorter. This corresponds to more efficient propagation of the elastic wave through the densely-packed media at the beginning of the phase 
transition when the structure does not contain microstructural defects. As the transition proceeds, the number of microcracks in the crystal interior increases. The defects dissipate the elastic strain energy and decrease the energy and amplitude of the subsequent acoustic signals. Accordingly, the velocity of the acoustic waves decreases, and the emission required longer time to reach maximal amplitude, as reflected in higher risetimes (Table S1, Supporting Information).
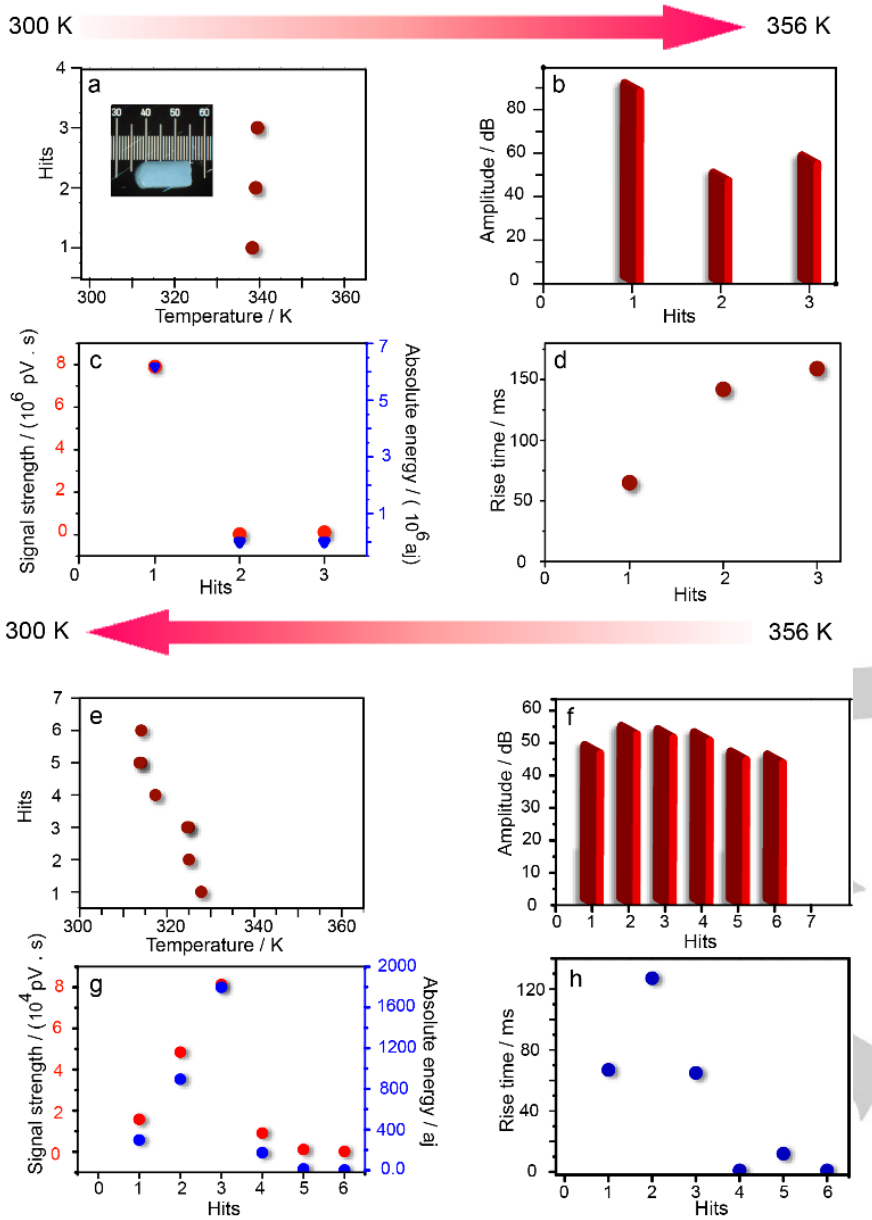

crystal recorded upon heating (a-

Figure 3. Acoustic emission from an L-PGA crystal recorded upon heating (a$\mathrm{d})$ and cooling ( $\mathrm{e}-\mathrm{h})$ over the thermosalient phase transition. The "hits" are
related to the progression of a disturbance as a result of rapid phase transition in a crystal domain in form of a longitudinal pressure wave (Figure 2a). The number of hits of a wave corresponds to the times the disturbance exceeds an arbitrary threshold value. A hit count is the number of times the pressure increases above the threshold. The signal strength is the intensity of each hit, the amplitude is the difference between the intensity of the strongest positive and strongest negative value of the signal, rise time is the time it takes for the signal since the first time it crosses the threshold to reach maximum intensity.

Compared to heating, cooling of the same crystal generated more acoustic emissions. The acoustic signals on cooling were also distributed in a wider temperature window, which extended beyond the hysteresis of the phase transition determined by calorimetry. When the temperature was decreased from $356 \mathrm{~K}$ to $300 \mathrm{~K}$, six distinct emission hits were detected in the temperature range $327.7-314 \mathrm{~K}$, although these were much lower in energy and intensity relative to the signal during heating (Figure $3 \mathrm{e}-\mathrm{h}$, Table S1, Supporting Information). Possible reasons for this observation are fragmentation of the parent crystal and the slightly different temperature of the reverse transition (from $\beta$ to $\alpha$ ) of the individual pieces that would result in distinct acoustic hits. Although L-PGA crystals are unusually robust ${ }^{2}$ the probability of fragmentation increases in crystals which have been restrained from motion by which they would otherwise relax, that is, by transducing the accumulated strain energy into kinetic energy of motion. However, this may not be the sole source of acoustic emission because signals were observed even at much lower temperature $(314 \mathrm{~K})$. Thus, structural relaxation and thermal contraction of the crystal lattice could also contribute to the generation of the acoustic signals during cooling. As supportive evidence of this argument, we found that racemic crystals of PGA, which remain immotile, ${ }^{2}$ also produce distinct emission hits during both heating and cooling, although the intensity of the signals was much lower than those from the chiral crystals (Figure S1 and Table S2, Supporting Information). Single crystals that did not disintegrate also generated acoustic emission. These observations indicate that the acoustic emission is generated during the transition, and not during the relaxation of the crystal that results in crystal explosion. The generality of the acoustic emission of thermosalient solids was confirmed with an earlier report on oxitropium bromide, ${ }^{5}$ and also with the crystals of another thermosalient compound, 1,2,4,5-tetrabromobenzene (Supporting Information, Figure S2 and Table S3). The crystals of this compound, which undergo thermosalient phase transiton at $315.3 \mathrm{~K}$ on heating and $305.8 \mathrm{~K}$ on cooling ${ }^{3}$ were also observed to generate distinct and detectable acoustic emission.
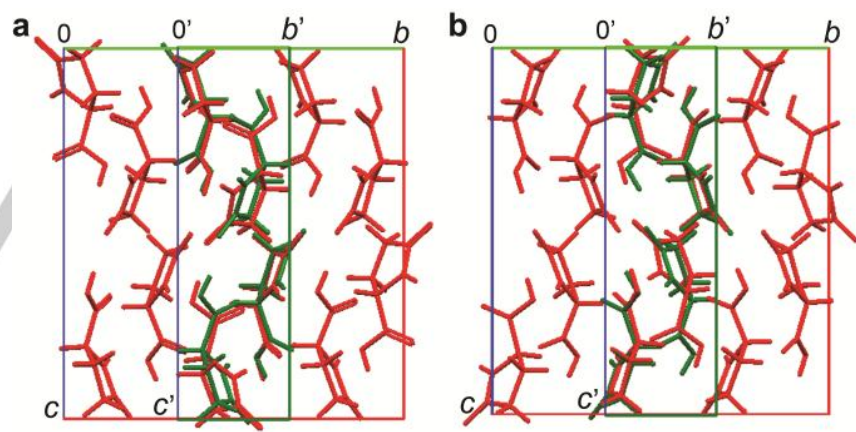

Figure 4. Overlapped packing in the crystals of the room-temperature phases $(\alpha)$ and the high-temperature phases ( $\beta$ ) of L-PGA (a) and D-PGA (b). (a) The structures of forms $\alpha$ and $\beta$ were determined at $300 \mathrm{~K}$ and $353 \mathrm{~K}$, respectively. (b) The structures of forms $\alpha$ and $\beta$ were determined at $300 \mathrm{~K}$ and $360 \mathrm{~K}$, respectively. The unit cell of form $\beta\left(a^{\prime}, b^{\prime}, c^{\prime}\right)$ is obtained from that of form $\alpha(a$, $b, c)$ by $a^{\prime}=b, b^{\prime}=a / 3, c=c^{\prime}$. The structural data were taken from ref. ${ }^{2}$

The structural aspects of the phase transition of PGA crystals were studied thoroughly. ${ }^{2}$ Figure 4 shows overlapped models of the crystal structures of the two isomers, and Table S4 in the Supporting Information summarizes the basic unit cell parameters. Along with the observations with other thermosalient solids, ${ }^{3}$ and contrary to the large structural change that can be anticipated from the dramatic mechanical effect, the overall structural difference in packing between the two forms ( $\alpha$ and $\beta$ ) of each isomer is unexpectedly small (Supporting Information Table S4). 
When the room-temperature form $(\alpha)$ transitions to the hightemperature phase $(\beta)$, after transformation of the unit cell, we find that the $a$ axis expands $<1.7 \%$, the $c$ axis expands $<0.6 \%$, and the $b$ axis shrinks $<1.6 \%$. As a result of the bidirectional expansion and unidirectional contraction, the overall change of the unit cell per formula unit is small $(\sim+0.53 \%$ in L-PGA and $\sim+0.86 \%$ in D$P G A)$. The small structural change appears to be common for all thermosalient solids; ${ }^{3}$ another example, 1,2,4,5tetrabromobenzene, is provided in the Supporting Information Figure S3.

The rapid and linear progression of the phase boundary (the habit plane) in PGA reaches an impressive speed of $2.8 \mathrm{~m} / \mathrm{s} .^{2}$ In another previously studied thermosalient material, the phase moves at $0.54 \mathrm{~m} / \mathrm{s}^{1}$ This speed by which the phase progresses through the crystal of the parent phase is on the order of thousands of times faster than other phase transitions, such as spin-crossover transitions. Altogether, the structural similarity between the phases, and the anisotropic thermal expansion preceding the transition are consistent with other thermosalient solids (for articles and reviews that discuss this topic, see refs. ${ }^{3,4,9,10}$ ), and lend strong experimental support to the martensitic character of the thermosalient phase transitions.

In the crystal of PGA, the molecules are held with each other by hydrogen bonds (Figure 4), and hence this compound, together with two other known examples 8,15 were classified as class III thermosalient solid. 3,4 1,2,4,5-Tetrabromobenzene which also generates acoustic waves (Supporting Information Figure S2 and S4, and Table S3) is a typical member of class I thermosalient solids, which are composed of oblate or cylindrical molecules that do not contain hydrogen-bonding groups and tend to pack in layers. ${ }^{7}$ Oxitropium bromide ${ }^{5}$ is one of the class II thermosalient solids that lack groups available for intermolecular hydrogen bonding and are decorated by a high number of bulky, flexible groups (note that oxitropium bromide does have an hydroxyl group however it forms an intramolecular hydrogen bond), the results cover all three classes of thermosalient materials (for more details on the classification, see references ${ }^{3,9,10}$ ). Within a broader perspective, the results reported here not only confirm that the acoustic emission is common for the thermosalient materials at their phase transition, but they also provide strong support to the earlier conclusions that these materials can be considered organic analogues of the inorganic martensites. ${ }^{1}$

In summary, we have demonstrated that the thermosalient phase transitions in organic-containing crystals are commonly accompanied with acoustic emission-a behavior that is atypical for molecular solids and resembles that of martensitic transitions in steel and other metallic alloys. Sudden relaxation of the elastic stress at the interface of the two phases leads to bursts of acoustic emission that carry information on the energetics, kinetics and hysteric behavior of the transition. Moreover, we observed that the kinetics of wave propagation depends on the packing homogeneity and microstructural defects in the crystal interior. This study provides a prospective direction for future use of acoustic emission techniques to directly study rapid events during the phase transition in organic martensites that are currently not accessible with other methods, and could provide better understanding of the related macroscopic dynamic events.

\section{Experimental Section}

Materials. Crystalline L-PGA (CAS no. 98-79-3) and D-PGA (4042-36-8) were obtained from Sigma-Aldrich and was used without purification. Racemic PGA (CAS no. 149-87-1) was also obtained from Sigma-Aldrich and was recrystallized from methanol. 1,2,4,5-tetrabromobenzene (CAS no. 636-28-2) was obtained from Aldrich and recrystallized from toluene. Acoustic emission. In a typical setup, the TS crystals were placed on a copper plate $\left(5 \times 5 \mathrm{~cm}^{2}\right)$ on top of the ceramic plate heater and covered lightly with another copper plate that served as a waveguide. A piezoelectric transducer with PZT (lead-zirconia-titanate) element (model $\mathrm{R} 3 \alpha$, from Mistras, operating frequency $25-70 \mathrm{kHz}$, input 4-20 mA, working temperature 208-448 K) was used as acoustic sensor and was attached to the heating copper plate with a thin layer of ultrasonic couplant (G, Sono-600, Sonotech Magnaflux). The sensor was connected to PC with a preamplifier (IL-LP-WS, band width $77-1100 \mathrm{kHz}$, Mistras) via an USB acoustic emission node (model 1283, Mistras; sampling frequency, $20 \mathrm{MHz}$ ). The sensor was not directly placed on top of crystal to avoid damage of the crystal. The ceramic heater was connected to a thermoelectric converter that was heated using Seebeck effect, while the temperature was measured by an internal K-type thermocouple (Omega, USA) which was fed to the heater controller. The heating and cooling rate was $10 \mathrm{~K} /$ minute. The sample temperature was measured with another thermocouple K-type sensor (Vernier) attached to the copper plate on which sample is seating and temperature values were logged into LabVIEW software program. The output acoustic parameters (hits, amplitude, rise time, energy) were collected as a function of temperature using custom modified LabVIEW software. The signal threshold amplitude was set to be $40 \mathrm{~dB}$. The experimental set up was fixed to vibration free table to decrease the background noise.

$X$-ray Powder Diffraction. The synchrotron X-ray powder diffraction (XRPD) patterns of L-PGA and D-PGA were collected in Debye-Scherrer mode at beamline P02.1 of the Petra III synchrotron of DESY (Hamburg) at an energy of $\sim 60 \mathrm{keV}(\lambda=0.2072 \AA)$ using an unfocused and collimated $X$-ray beam with size of $\sim 1.0 \times 1.0 \mathrm{~mm}^{2}$. The diffracted $\mathrm{X}$-rays were detected using a XRD1621 Perkin Elmer amorphous silicon image plate detector. The powder samples were placed in a quartz capillary of $0.8 \mathrm{~mm}$ diameter). For cooling/heating, a cold air blower (Oxford Cryostream 700) was mounted perpendicular to the X-ray beam axial to the capillary. The following heating and cooling scheme was applied for both samples: 295$325 \mathrm{~K}$ (10 K/scan), 325-349 K (2 K/scan), 349-315 K (-2 K/scan), 315$145 \mathrm{~K}(-10 \mathrm{~K} / \mathrm{scan}), 145-125 \mathrm{~K}$ (-2 K/scan), 125-155 K (+2 K/scan), 155$325 \mathrm{~K}(+10 \mathrm{~K} / \mathrm{scan})$, and $325-355$ (2 K/scan). In order to get reasonable time resolution and counting statistics, the measurement time was fixed to $180 \mathrm{~s} / \mathrm{scan}$ (107 patterns were recorded until the final temperature was reached). A complete run lasted 10.5 hours. The collected twodimensional Debye-Scherrer rings were subsequently integrated with the program FIT2D 44 to one-dimensional powder diffraction patterns. Parameters for integration were determined from $\mathrm{L} \mathrm{LaB}_{6}$ reference sample. The $2 \mathrm{D}$ and $3 \mathrm{D}$ plots created with the Powder $3 \mathrm{D}$ program ${ }^{45}$ show all powder patterns of a run is dependence on scattering angle and time.

\section{Acknowledgements}

This work was financially supported by NYU Abu Dhabi. P.N. thanks the Alexander von Humboldt foundation for the Friedrich Wilhelm Bessel 
Research Award. Parts of this research were carried out at the light source PETRA III at DESY, a member of the Helmholtz Association (HGF). We thank Dr. Nikolas Giakoumidis for his help in custom design of the experiment and LabVIEW software, and Dr. Durga P. Karothu for his help with the graphics.

Keywords: Acoustic emission $•$ Martensitic phase transition $•$ Mechanical effects • Organic crystal $\cdot$ Thermosalient effect

1. M. K. Panda, T. Runčevski, S. C. Sahoo, A. A. Belik, N. K. Nath, R E. Dinnebier, P. Naumov, Nat. Commun. 2014, 4811.

2. M. K. Panda, T. Runčevski, A. Hussain, R. E. Dinnebier, P. Naumov, J. Am. Chem. Soc. 2015, 137, 1895-1902.

3. S. C. Sahoo, M. K. Panda, N. K. Nath, P. Naumov, J. Am. Chem Soc. 2013, 135, 12241-12251.

4. N. K. Nath, M. K. Panda, S. C. Sahoo, P. Naumov, CrystEngComm 2014, 16, 1850-1858.

5. Ž. Skoko, S. Zamir, P. Naumov, J. Bernstein, J. Am. Chem. Soc. 2010, 132, 14191-14202.

6. S. Ghosh, M. K. Mishra, S. Ganguly, G. R. Desiraju, J. Am. Chem. Soc. 2015, 137, 9912-9921.

7. S. C. Sahoo, S. B. Sinha, M. S. R. N. Kiran, U. Ramamurty, A. F Dericioglu, C. M. Reddy, P. Naumov, J. Am. Chem. Soc. 2013, 135 13843-13850

8. M. K. Panda, R. Centore, M. Causà, A. Tuzi, F. Borbone, P. Naumov, Sci. Rep. 2016, 6, 29610.

9. P. Naumov, S. Chizhik, M. K. Panda, N. K. Nath, E. Boldyreva Chem. Rev. 2015, 115, 12440-12490.

10. P. Commins, I. T. Desta, D. P. Karothu, M. K. Panda, P. Naumov, Chem. Commun. 2016, 52, 13941-13954.

11. P. Naumov, S. C. Sahoo, B. A. Zakharov, E. Boldyreva, Angew. Chem. Int. Ed. 2013, 52, 9990-9995.

12. R. Medishetty, A. Husain, J. Bai, T. Runčevski, R. E. Dinnebier, P. Naumov, J. J. Vittal, Angew. Chem. Int. Ed. 2014, 53, 5907-5911.

13. R. Medishetty, S. C. Sahoo, C. E. Mulijanto, P. Naumov, J. J. Vittal, Chem. Mater. 2015, 27, 1821-1829.

14. T. Seki, K. Sakurada, M. Muromoto, H. Ito, Chem. Sci. 2015, 6 1491-1497.

15. D. P. Karothu, J. Weston, I. T. Desta, P. Naumov, J. Am. Chem. Soc. 2016, 138, 13298-13306.

16. A. L. Roitburd, G. V. Kurdjumov, Mat. Sci. Eng. 1979, 39, 141-167.

17. P. M. Kelly, J. Nutting, Proc. R. Soc. London A, 1960, 259, 45-58.

18. T. D. Kubyshkina, L. M. Pevzner, Y. M. Potak, Met. Sci. Heat Treat 1960, 2, 425-430.

19. A. G. Crocker, B. A. Bilby, Acta. Metall. 1961, 9, 678-688.

20. X. Huang, G. J. Ackland, K. M. Rabe, Nat. Mater. 2003, 2, 307-311.
21. J. Cui, Y. S. Chu, O. O. Famodu, Y. Furuya, J. Hattrick-Simpers, R. D. James, A. Ludwig, S. Thienhaus, M. Wuttig, Z. Zhang, I. Takeuchi, Nat. Mater. 2006, 5, 286-290.

22. H. Y. Kim, Y. Ikehara, J. Y. Kim, H. Hosoda, S. Miyazaki, Acta Mater 2006, 54, 2419-2429.

23. D. Stroz, D. Chrobak, Mater. Trans. 2011, 52, 358-363.

24. M. Nishida, T. Hara, T. Ohba, K. Yamaguchi, K. Tanaka, K, K. Yamauchi, Mater. Trans. 2003, 44, 2631-2636.

25. P. M. Giles, M. H. Longenbach, A. R. Marder, J. Appl. Phys. 1971, $42,4290-4295$.

26. J. A. KrumhansI, Y. Yamada, Mater. Sci. Eng. A 1990, 127, 167181.

27. M. H. Bocanegra-Bernal, S. D. De la Torre, J. Mater. Sci., 2002, 37, 4947-4971.

28. V. D. Blank, B. A. Kulnitskiy, Scripta Mater. 1997, 37, 313-316.

29. W. P. Mason, D. N. Beshers, M. C. Jon, J. T. Kuo, Ultrasonics, 1975 13, 128-131.

30. J. W. Laughner, T. W. Cline, R. E. Newnham, L. E. Cross, Phys Chem. Min. 1979, 4, 129-137.

31. J.-B. Chung, E. Kannatey-Asibu, J. Appl. Phys. 1992, 72, 1812 1820.

32. K. Ono, H. Cho, M. Takuma, J. Acoustic Emis. 2005 23, 206-214.

33. V. R. Skal's'kyi, O. E. Andreikiv, O. M. Serhienko, Mater. Sci. 2003 39, 86-107.

34. M. Janeček, R. Kral, P. Dobroň, F. Chemelik, V. Supik, F. Holländer Mater. Sci. Eng A, 2007, 462, 311-315.

35. E. Brevel, V. Srikanth, E. C. Subbarao, J. Am. Ceram. Soc. 1995 $782541-2544$

36. V. Srikanth, E. C. Subbarao, G. V. Rao, Ceram. Int. 1992, 18, $251-$ 259.

37. E. A. Dul'kin, I. P. Raevski, S. M. Emelyanov, Phys. Solid State, 1997, 39, 316-317.

38. T. Sawada, H. Gohshi, C. Abe, K. Furuya, Anal. Chem. 1985, 57, 1743-1745.

39. O. E. Martinez, Y. Cesa, N. Mingolo, R. Romerio, Appl. Phys. B, 2005, 80, 365-371.

40. P. W. J. Glover, P. Baud, M. Darot, P. G. Meredith, S. A. Boon, M. LeRavaleq, S. Zoussi, T. Reuschle, Geophys. J. Int. 1995, 120, 775-782.

41. K. Prabakar, S. P. M. Rao, Ferroelectric Lett. 2005, 32, 99-110.

42. C. Meade, R. Jeanloz, Nature, 1989, 339, 616-618.

43. R. K. Vasudevan, H. Khassaf, Y. Cao, S. Zhang, A. Tselev, B Carmichael. M. B. Okatan. L-Q. Chen, S. P. Alpay, S. V. Kalinin, N. Bassiri-Gharb, Adv. Funct. Mater. 2016, 26, 478-486.

44. A. P. Hammersley, S. O. Svensson, M. Hanfland, A. N. Fitch, D Hausermann, High Pressure Res. 1996, 14, 235-248.

45. B. Hinrichsen, R. E. Dinnebier, M. Z. Jansen, Z. Krist. 2006, 23 231-236. 


\section{Entry for the Table of Contents}

\section{COMMUNICATION}

Text for Table of Contents

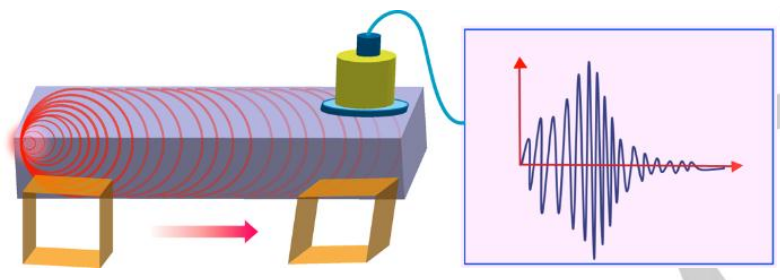

Manas K. Panda, Martin Etter, Robert E. Dinnebier, Panče Naumov *

Page No. - Page No.

Acoustic Emission from Organic Martensites 